\title{
Effets de l'acide trans-10, cis-12 linoléique sur le métabolisme des lipides dans le foie chez la souris C57BL/6
}

\author{
Pascal DEGRACE ${ }^{1}$ \\ Laurent DEMIZIEUX ${ }^{1}$ \\ Ismaël MOHAMED ${ }^{1}$ \\ Joseph GRESTI ${ }^{1}$ \\ Jean-Michel CHARDIGNY² \\ Jean-Louis SÉBÉDIO² \\ Pierre CLOUET ${ }^{1}$ \\ ${ }^{1}$ UPRES Lipides et nutrition \\ Faculté des sciences Gabriel, 21000 Dijon \\ 2 INRA, Unité de nutrition lipidique, \\ 21034 Dijon Cedex
}

\begin{abstract}
A 4-week diet containing 1\% of the conjugated trans-10, cis-12 linoleic acid (CLA2) induces, in C57BL/6 mice, a lipodystrophy, a liver steatosis and a reduction of plasma triglycerides. We demonstrate that, first, hepatic lipid accumulation is not due to an in vivo alteration of lipoprotein production. Fates of CLA2 relative to ß-oxidation reactions are then studied in mitochondrial fractions isolated from liver of control animals and the data indicate not only that the CLA is poorly oxidised but also that it modifies the oxidation of usual fatty acids. We also demonstrate that oxidation capacities are increased by CLA2 treatment concomitantly to carnitine palmitoyltransferase I (CPT I) activity. Besides, the increases in acetyl-COA carboxylase activity and malonyl-COA concentration in the liver of CLA2 treated mice provide evidence of a high rate of lipogenesis. The increased CPT I sensibility to malonyl-COA inhibition due to the treatment suggests that the whole fatty acid B-oxidation is finally reduced in vivo. The mechanisms implicated in the setup of the hepatic steatosis and in the reduction of the lipaemia despite the increased VLDL secretion are discussed.
\end{abstract}

Key words: conjugated linoleic acid, $\beta$-oxidation, carnitine, palmitoyltransferase I, liver steatosis, lipoprotein
Le terme CLA désigne un groupe $d^{\prime}$ isomères positionnels et géométriques de l'acide linoléique produits par hydrogénation et isomérisation bactériennes dans le rumen des ruminants ou par suite de traitements technologiques. II existe ainsi 28 formes possibles de CLA pour lesquels les doubles liaisons peuvent être en positions 7 à 13 le long de la chaîne de 18 carbones. La forme cis-9, trans-11 C18:2 (CLA1) représente l'isomère naturel le plus souvent rencontré dans les produits laitiers et carnés issus de ruminants, dans lesquels il peut représenter entre 0,5 et $2 \%$ des acides gras (AG) totaux. Les CLA disponibles dans le commerce sont généralement constitués d'un mélange d'isomères composé, entre autres, de $40 \%$ de CLA1 et de $40 \%$ d'acide trans-10, cis-12 C18:2 (CLA2). La production naturelle de CLA2 est très faible et la présence de cet isomère dans l'organisme est essentiellement le résultat d'une absorption directe de la molécule générée par traitements technologiques et culinaires.

Les effets biologiques attribués aux CLA sont nombreux, souvent mal compris, voire contradictoires. Les CLA sont décrits comme des modulateurs de la réponse immunitaire [1, 2], comme possédant des propriétés anticancéreuses $[3,4]$, anti-athérogéniques $[5,6]$, antidiabétiques $[7,8]$ ou encore comme responsables d'une augmentation de la résistance à I'insuline $[9,10]$. L'effet réducteur des CLA sur la masse grasse décrit dans plusieurs modèles animaux [11-14] apparaît très spectaculaire, même si les études menées chez l'Homme ne sont pas aussi démonstratrices $[15,16]$. Quoi qu'il en soit, la multiplicité des modèles d'études et l'utilisation de mélanges d'isomères de
CLA rendent les interprétations délicates quant aux effets réels de chacun des isomères. L'utilisation d'isomères purifiés a permis de montrer que les effets des CLA sur les paramètres lipidiques, déjà évoqués dans d'autres études réalisées avec des mélanges d'isomères $[17,18,2]$, sont en fait majoritairement attribuables à la forme trans-10, cis-12 (CLA2) et c'est pourquoi cet article sera essentiellement consacré à cet isomère.

\section{Effets du CLA2 sur les paramètres lipidiques chez la souris}

Une sévère régression de la masse adipeuse et une stéatose hépatique sont observées lorsque des souris C57BL/6 âgées de 7 semaines sont soumises à un régime standard enrichi avec
$1 \%$ de CLA2 pendant 4 semaines (tableau 1). Les animaux présentent également une baisse de $70 \%$ des triglycérides plasmatiques, qui semble correspondre à une diminution de la concentration plasmatique en VLDL à en juger par la diminution de la concentration en apoB.

De nombreuses pathologies sont associées à une stéatose hépatique. On peut évoquer I'alcoolisme, l'obésité, le diabète, les défauts génétiques, les hépatites, les intoxications... Quoi qu'il en soit, l'infiltration du foie par les graisses ne peut avoir lieu que s'il existe une perturbation de l'homéostasie lipidique hépatique. Parmi les causes possibles figurent : 1) un défaut de sécrétion des lipoprotéines, comme cela est le cas, par exemple, chez la souris Suncus [19] ; 2) une augmentation de l'entrée des AG par suite d'un captage accru des lipides plasmatiques; 3) une augmentation de la

Tableau 1. Effets du CLA2 sur la masse des souris, du foie, du tissu adipeux et sur des paramètres lipidiques sanguins (d'après [20]).

\begin{tabular}{|lcc|}
\hline & Témoin & CLA2 \\
\hline Masse corporelle (g) & $25,68 \pm 0,87$ & $23,29 \pm 0,56^{*}$ \\
Foie & & \\
$\quad$ Masse relative (\% masse corporelle) & $4,67 \pm 0,11$ & $7,53 \pm 0,29^{*}$ \\
$\quad$ Triacylglycérols (mg/g de tissu) & $8,55 \pm 1,62$ & $64,92 \pm 6,68^{*}$ \\
$\begin{array}{l}\text { Tissu adipeux périépididymaire } \\
\quad \text { Masse relative (\% masse corporelle) }\end{array}$ & $1,18 \pm 0,07$ & $0,17 \pm 0,06^{*}$ \\
$\begin{array}{l}\text { Plasma } \\
\quad \text { Triacylglycerols (g/l) }\end{array}$ & $1,04 \pm 0,15$ & $0,34 \pm 0,03^{*}$ \\
ApoB (g/l) & $0,081 \pm 0,012$ & $0,038 \pm 0,005^{*}$ \\
\hline
\end{tabular}

Des souris C57BL6j âgées de 7 semaines sont soumises à un régime standard enrichi avec $1 \%$ de C18 :1n-9 (lot témoin) ou de CLA2 pendant 4 semaines. Le CLA2 purifié est administré sous forme de triglycérides. Al'issue de la période de traitement, les animaux sont sacrifiés à l'état nourri. ${ }^{*} \mathrm{P}<0,05$. 
lipogenèse et 4) une diminution de l'oxydation des AG.

\section{Effets sur la sécrétion des lipoprotéines par le foie}

Des expériences réalisées sur des lignées cellulaires de foie humain (HepG2) ont montré que les CLA avaient un effet inhibiteur sur la sécrétion des VLDL [21, 22], ce qui conduisait à interpréter l'effet hypolipémiant des CLA observé dans plusieurs études [5, 23-25, 18, 26] comme le résultat d'une diminution de la sécrétion hépatique de lipoprotéines. Toutefois, la mesure in vivo des capacités du foie à sécréter les lipoprotéines a indiqué au contraire que les voies métaboliques impliquées dans la synthèse et la libération des VLDL hépatiques sont parfaitement fonctionnelles et que les capacités sont même augmentées chez les souris traitées par le CLA2 (figure 1). Par conséquent, la stéatose hépatique induite par le CLA2 ne peut résulter d'un défaut d'assemblage ou de libération des lipoprotéines par le foie.

\section{Effets sur la ß-oxydation des acides gras à longue chaîne (AGLC) dans le foie}

\section{B-oxydation mitochondriale du CLA2}

Une étude concernant l'oxydabilité des CLA via les voies métaboliques empruntées depuis la traversée de la membrane mitochondriale jusqu'à la chaîne respiratoire a révélé que : 1) les CLA sont de médiocres substrats pour les réactions de ß-oxydation; 2) les CLA sont capables de perturber les réactions mitochondriales de la ß-oxydation d'AG courants comme le palmitate ou le palmitoléate; 3 ) l'altération de l'oxydation des AG par les CLA serait due en partie à une activité élevée du transfert de ces isomères à travers la membrane interne. En résumé, l'ensemble des données semble indiquer que les CLA traversent particulièrement bien la membrane mitochondriale et, comme leur conformation particulière est susceptible de perturber certaines étapes de ß-oxydation (enzymes auxiliaires), ils pourraient s'accumuler dans la matrice et provoquer un ralentissement de l'oxydation de I'ensemble des AG (figure 2).

Un ralentissement des réactions de ß-oxydation pourrait favoriser l'accumulation de graisses mais également augmenter la disponibilité des AGLC et des CLA pour d'autres voies métaboliques. Ainsi, une proportion significativement plus faible de CLA2 que de CLA1 est observée dans le foie et le tissu adi-

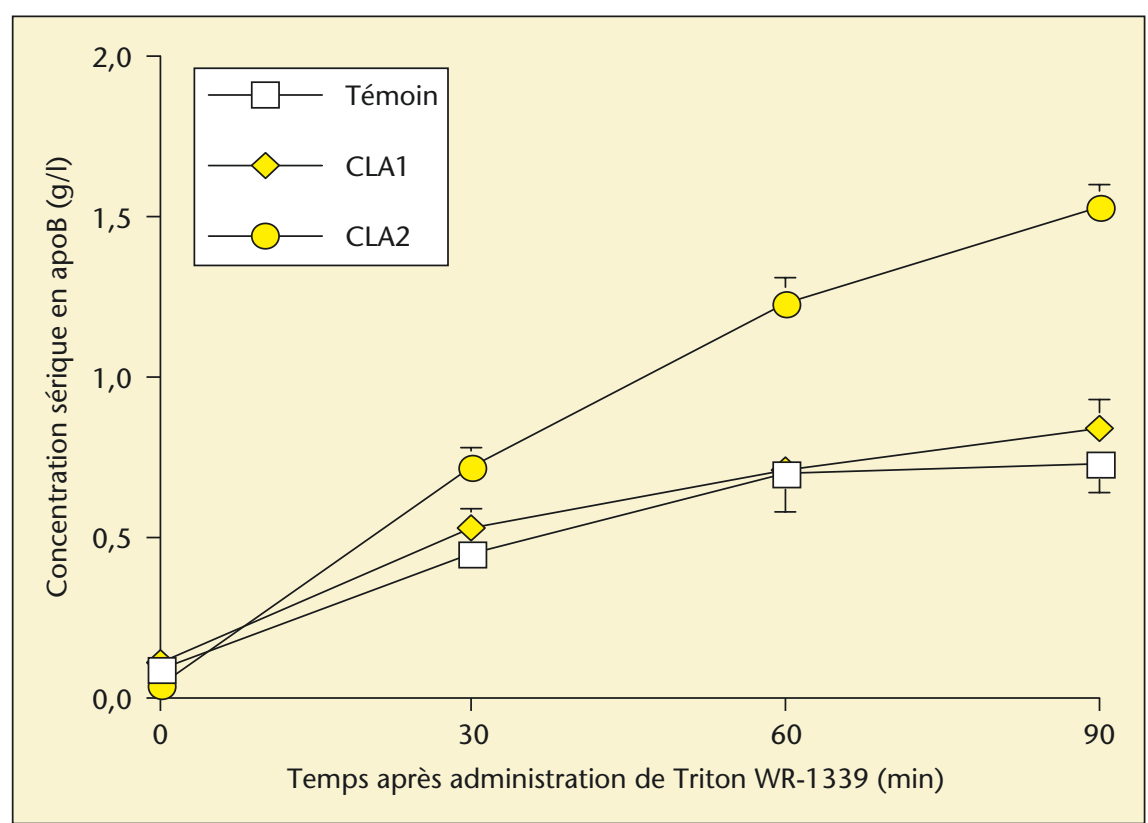

Figure 1. Effets des deux principaux CLA sur les capacités du foie à sécréter les VLDL-apoB. Une injection intraveineuse de Triton WR-1339 bloque la dégradation des lipoprotéines plasmatiques par inhibition de l'activité de la lipoprotéine lipase et provoque l'accumulation dans le sang des VLDL sécrétées par le foie. La concentration sérique en VLDL est déterminée en dosant les apoB par immuno-turbidimétrie à partir d'échantillons prélevés toutes les 30 minutes pendant $90 \mathrm{~min}$.

peux de souris à qui des quantités équivalentes de chacun des isomères ont été administrées. Le CLA2 étant un bon substrat pour les réactions d'élongation/désaturation [27] et pour la

production d'eicosanoïdes [28], il est tout à fait envisageable que des métabolites du CLA2 soient à l'origine de certains des effets attribués à cet isomère.

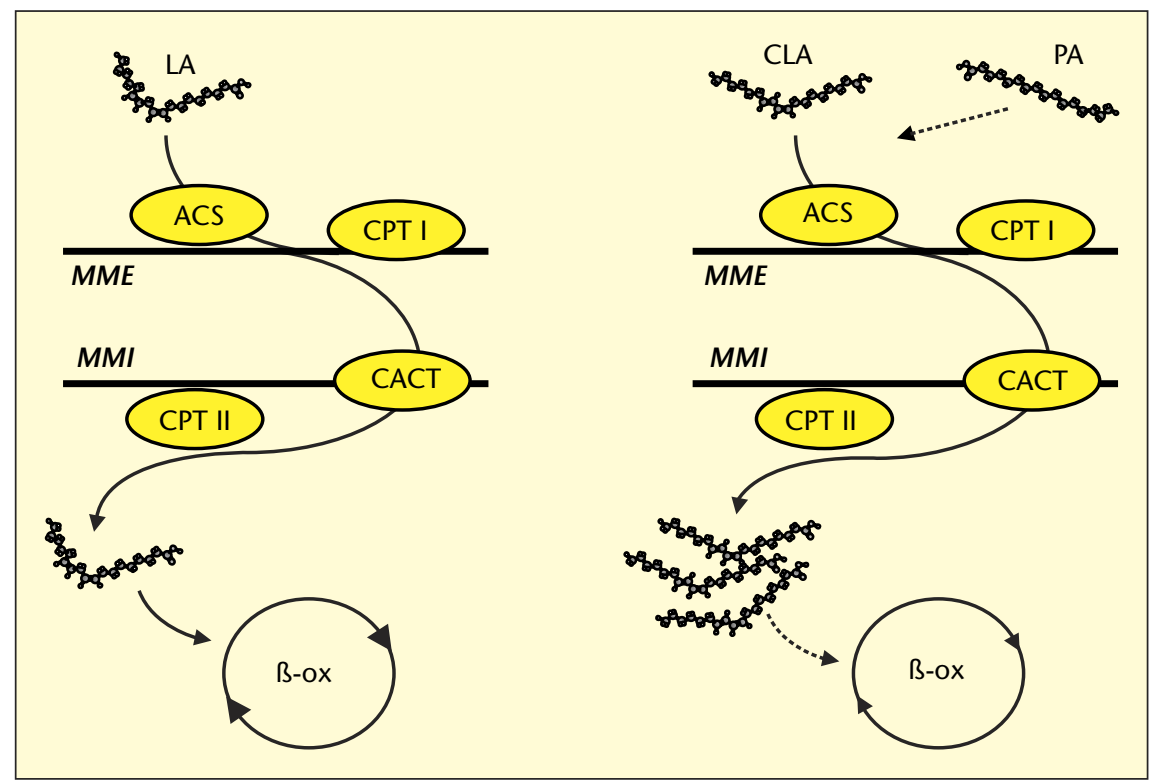

Figure 2. Mécanisme hypothétique du ralentissement de la ß-oxydation des acides gras à longue chaîne par les CLA. ACS, acyl-CoA synthétase; $\beta$-ox, $B$-oxydation ; CACT, carnitine acylcarnitine transférase ; CLA, acides cis-9, trans- 11 ou trans-10 cis-12 linoléique; CPT I, carnitine palmitoyltransférase I; CPT II, carnitine palmitoyltransférase II ; LA, acide linoléique; $M M E$, membrane mitochondriale externe; $M M I$, membrane mitochondriale interne; $P A$, acide palmitoléique. 
Tableau 2. Concentrations en carnitine, activités mitochondriales et péroxisomales relatives à l'oxydation des acides gras dans les foies de souris traitées par le CLA2 (d'après [29]).

\begin{tabular}{|lcc|}
\hline & Témoin & CLA2 \\
\hline $\begin{array}{l}\text { Carnitine totale (nmol/g tissu frais) } \\
\text { Activités mitochondriales }\end{array}$ & $119 \pm 10$ & $126 \pm 8$ \\
$\quad$ - CPT I (nmol/min.mg protéine. mitochondriale ${ }^{-1}$ ) & $2,12 \pm 0,18$ & $2,69 \pm 0,14^{*}$ \\
$\quad$ - Oxydation du palmitate & & \\
$\quad$ (nmol/min.mg tissu frais ${ }^{-1}$ ) & $380 \pm 64$ & $573 \pm 10^{*}$ \\
$\quad$ (nmol/min.mg protéine. ${\text { mitochondriale }{ }^{-1} \text { ) }}^{\text {Activités péroxisomales }}$ & $4,45 \pm 0,35$ & $5,47 \pm 0,15^{*}$ \\
$\quad$ - PFAOS (nmol/min.g tissu frais ${ }^{-1}$ ) & & \\
$\quad$ - Oxydation du palmitate (nmol/min.g tissu frais ${ }^{-1}$ ) & $1,60 \pm 0,14$ & $2,97 \pm 0,24^{*}$ \\
\end{tabular}

Les valeurs indiquées correspondent à des moyennes \pm SEM $(n=5)$ obtenues à partir de tissu frais, d'homogénat tissulaire ou de mitochondries de foie. L'activité PFAOS (Peroxisomal Fatty Acid Oxidizing System) est déterminée en mesurant le NADH formé dans les premières étapes de la ß-oxydation péroxysomale. L'oxydation péroxisomale du palmitate est mesurée à partir d'un homogénat de foie en présence d'inhibiteurs de l'oxydation mitochondriale. ${ }^{*} \mathrm{P}<0,05$.

\section{Capacités $B$-oxydatives des foies d'animaux traités par le CLA2}

La fonctionnalité et les capacités des systèmes de dégradation des AGLC, ß-oxydations mitochondriale et péroxysomale, ont été testées in vitro à partir d'homogénats de foie et de mitochondries isolées. Les données présentées dans le tableau 2 indiquent que, pour des concentrations physiologiques en carnitine, les taux d'oxydation du palmitate sont toujours plus élevés dans les homogénats et les fractions mitochondriales de foies de souris traitées par le CLA2. Ce résultat peut être directement corrélé à l'augmentation de l'activité de la carnitine palmitoyltransférase I (CPTI), enzyme limitante du système de transport des AGLC à travers la membrane mitochondriale, et des ARNm de ses isoformes hépatique ( $L$ ) et musculaire (M). Les activités relatives à l'oxydation péroxysomale (Peroxisomal Fatty Acid Oxidizing System, PFAOS) sont également augmentées tandis que les ARNm de l'acyl-CoA oxydase sont induites par le CLA2.

Quoi qu'il en soit, une augmentation de l'activité oxydative, mesurée in vitro, peut ne pas refléter la réalité physiologique et peut même, au contraire, traduire un déficit oxydatif comme cela était le cas dans le foie de rats déficients en carnitine [30]. Ainsi, pour tenter d'évaluer les activités oxydatives in vivo, il est indispensable de prendre en considération les étroites relations qui lient les voies de la lipogenèse à celles de l'oxydation.

\section{Effets du CLA2 sur la lipogenèse et conséquences sur les taux d'oxydation in vivo}

Dans le foie des souris $C L A 2$, les augmentations de l'activité acétyl-CoA carboxylase (ACC) CLA2 (d'après [29]) de l'expression des ARNm de la fatty acid synthase (FAS) observée par Clément et al. [31] chez des souris traitées par le CLA2 suggère également une augmentation de la lipogenèse chez ces animaux.

Malgré l'augmentation des capacités oxydatives observée dans le foie des souris traitées, les données concernant la sensibilité de la CPT I au malonyl-CoA (figure 3) suggèrent, au contraire, qu'une inhibition du catabolisme oxydatif des AG s'exerce in vivo. En effet, la concentration élevée en malonyl-CoA associée à la forte sensibilité de la CPT I observée chez les souris traitées doivent conduire à une déviation des AG vers les voies d'estérification. De plus, les augmentations d'activité et d'expression de la CPT I mesurées chez les souris exposées au CLA2 plaident paradoxalement en faveur d'une réduction de l'oxydation in vivo puisque de telles inductions interviennent généralement dans des conditions physiologiques inhibitrices (à l'état nourri) ou pharmacologiques [30].

Les effets du CLA2 sur les voies de synthèse, de catabolisme et d'exportation des AG dans le foie sont représentés dans la figure 4 ; ces effets sont caractéristiques d'un état hyperinsuliné-

Tableau 3. Activité acétyl-CoA carboxylase et concentration en malonyl-CoA dans les foies de souris traitées par le

\begin{tabular}{|lcc|}
\hline & Témoin & CLA2 \\
\hline Activité acétyl-CoA carboxylase (nmol/min.g tissu frais ${ }^{-1}$ ) & $215 \pm 13$ & $328 \pm 9^{*}$ \\
Concentration en malonyl-CoA (nmol/g tissu frais) & $88 \pm 11$ & $144 \pm 9^{*}$ \\
\hline
\end{tabular}

Les valeurs indiquées correspondent à des moyennes $\pm \operatorname{SEM}(n=5) .{ }^{*} P<0.05$.

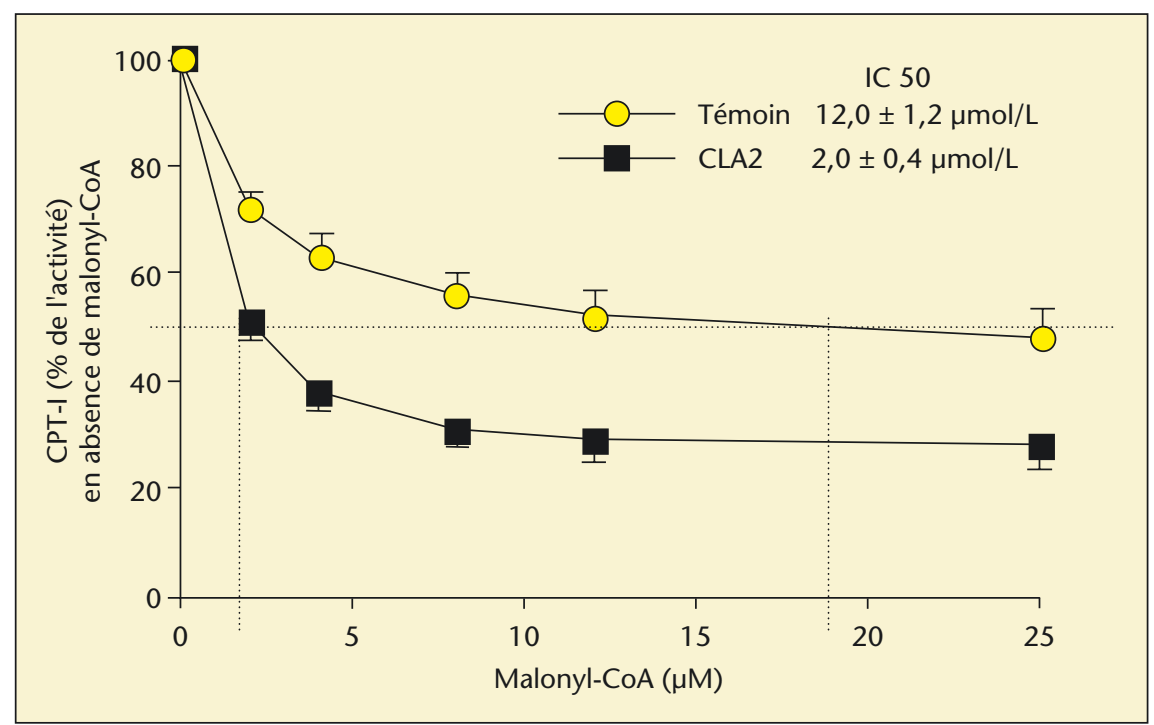

Figure 3. Sensibilité de la CPT I à l'inhibition par le malonyl-COA dans des mitochondries de foies de souris traitées par le CLA2. Les valeurs indiquées correspondent à des moyennes \pm SEM $(n=5)$ et sont exprimées en pourcentage des activités CPT I obtenues pour une concentration en malonyl-CoA égale à 0. L'encart «[IC 50] » indique, pour chaque série, les valeurs des concentrations en malonyl-CoA réduisant de $50 \%$ l'activité CPT I. 


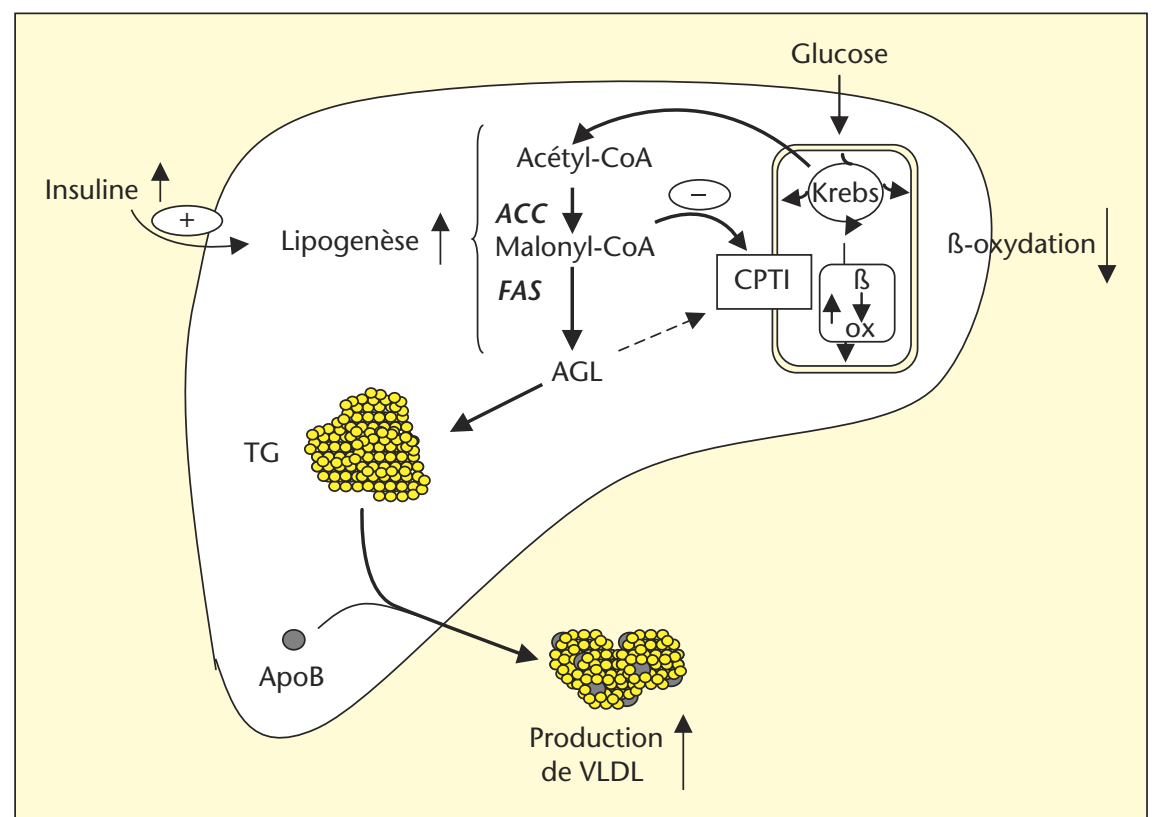

Figure 4. Modifications métaboliques intervenant dans I'hépatocyte de souris soumises à un régime contenant du CLA2. ACC, acétyl-COA carboxylase ; ACL, acide gras libre ; CPT I, carnitine palmitoyltransférase I; FAS, fatty acid synthase; TG, triacylglycérol.

mique avec, toutefois, deux évènements concomitants inhabituels: une réduction des lipides plasmatiques et une fonte du tissu adipeux.

\section{Mécanismes pouvant conduire à la baisse de la lipémie par le CLA2}

L'hypothèse d'une augmentation de la clairance des lipides sanguins par les tissus extrahépatiques a été envisagée pour tenter d'expliquer le paradoxe existant entre l'augmentation de la sécrétion des lipoprotéines par le foie des souris traitées et la baisse de la lipémie. Toutefois cette possibilité est peu vraisemblable, compte tenu de la fonte du tissu adipeux qui constitue habituellement un lieu privilégié de stockage des graisses, et de l'activité lipoprotéine lipase (LPL) inchangée dans le cœur [20] qui est pourtant un organe dont les AG représentent le principal substrat énergétique.

Des expérimentations menées sur des souris traitées pendant 15 et 28 jours par le CLA2 ont montré que l'augmentation de la masse du foie et la fonte du tissu adipeux dépendent de la durée du régime (données non présentées). L'analyse des lipides sanguins est beaucoup plus inattendue puisque la triglycéridémie est fortement augmentée après 15 jours de régime CLA2, alors qu'elle devient plus faible que celle des témoins à 28 jours. Les profils lipoprotéiques indiquent que c'est à la seule fraction VLDL qu'incombent les variations de concentration en triglycérides plasmatiques.
La production de VLDL par le foie est fortement liée à la quantité d'AC alimentant la voie de biosynthèse des lipoprotéines [33]. Les sources majeures d'AG pour les hépatocytes sont les AG libres circulants issus de la lipolyse adipocytaire, ceux issus de I'hydrolyse des TG constitutifs des lipoprotéines et les lipoprotéines elles-mêmes, chacune d'entre elles étant capable de stimuler la sécrétion des VLDL [34, 35].

L'induction de l'expression des ARNm des récepteurs aux LDL (LDLr) et aux VLDL (VLDLr) dans le foie des souris CLA2 suggère une augmentation de la prise en charge des lipides plasmatiques par cet organe lui-même [29]. Le VLDLr, peu exprimé dans le foie des animaux appartenant au groupe témoin, est fortement induit chez les souris traitées 15 jours et 28 jours par le CLA2. La relation entre le niveau d'expression du VLDLr et les taux de triglycérides plasmatiques a déjà été mentionnée dans d'autres études; en effet, des souris VLDLrdéficientes ou surexprimant le gène présentent, lorsqu'elles sont soumises à un régime hyperlipidique ou à un jeûne prolongé, respectivement une hausse ou au contraire une baisse des triglycérides plasmatiques [36]. De plus, il a été montré que la surexpression du VLDLr dans

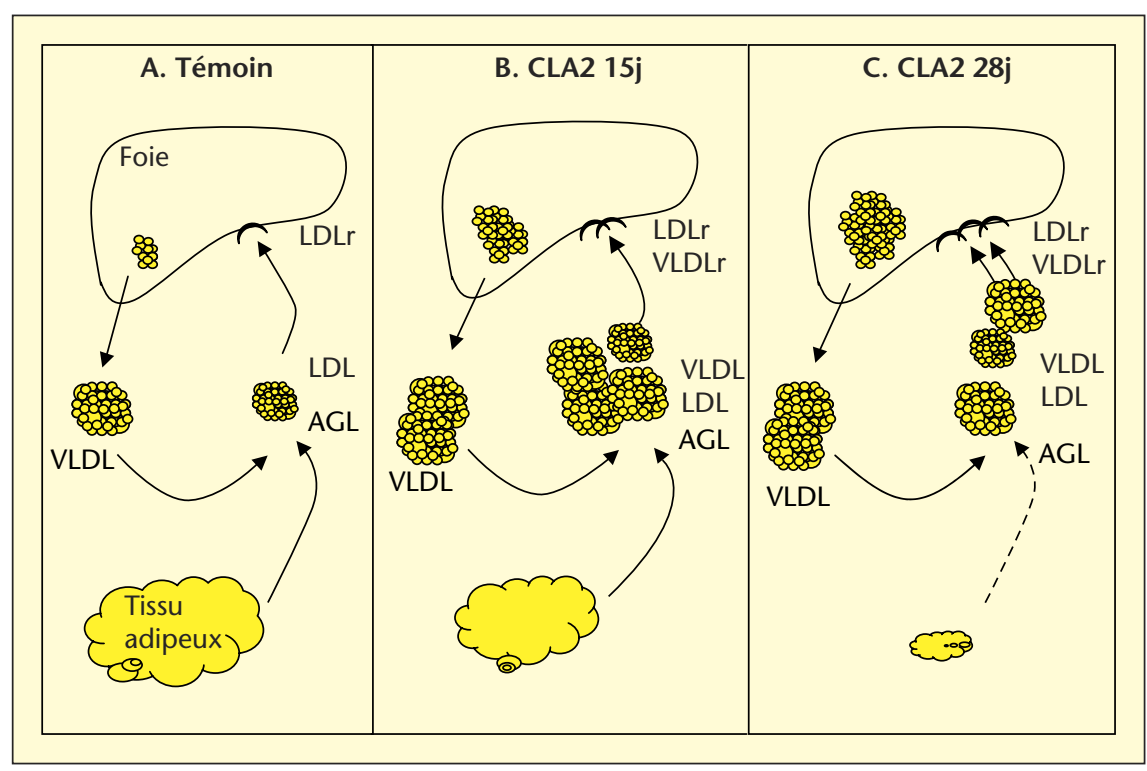

Figure 5. Schéma illustrant l'hypothèse de l'implication des récepteurs hépatiques et du tissu adipeux dans la baisse de la lipémie observée chez des souris traitées 28 jours par le CLA2.

A. Chez les témoins, les VLDL sécrétées sont hydrolysées par les tissus extrahépatiques et les résidus sont captés par le foie via les récepteurs aux $L D L$. B. Après 15 jours de régime, les lipides non stockés, les $A G$ libres libérés du tissu adipeux par la lipase hormono-sensible et ceux issus de la lipogenèse hépatique alimentent la voie de synthèse des VLDL dont la concentration augmente dans le plasma. Bien qu'on assiste à une induction de l'expression de protéines impliquées dans la prise en charge des lipides par le foie, les capacités de captage sont insuffisantes et la triglycéridémie augmente. C. Après 28 jours de traitement, le tissu adipeux ayant quasi disparu, les AG libres libérés par hydrolyse des TG stockés sont diminués. Les AG libres constituant une source pour la voie de synthèse des VLDL, ces dernières sont sécrétées en moins grand nombre. Même si les VLDL sont libérées encore plus abondamment que chez les témoins, la capacité des hépatocytes à capter les lipides plasmatiques est maintenant supérieure au flux de lipides arrivant au foie, ce qui aboutit à une baisse de la lipémie mais provoque aussi le développement de la stéatose hépatique. 
le foie pouvait corriger l'hypercholestérolémie [37] et fortement induire la clairance des IDL et LDL plasmatiques chez des souris déficientes en LDLr [38]. Ainsi, le fait que le foie stéatosé des souris traitées par le CLA2 surexprime le VLDLr par rapport aux témoins laisse supposer que ce récepteur participe à la prise en charge des lipoprotéines plasmatiques et, en particulier, des VLDL qui sont présentes en concentrations élevées.

Sous l'effet du traitement CLA2, on assiste à une arrivée massive au foie de lipides qui ne peuvent plus être stockés par le tissu adipeux et à une induction de l'expression des LDLr et VLDLr. La baisse de la triglycéridémie observée après 28 jours de traitement par le CLA2 est à rapprocher d'une part de l'augmentation des capacités de captage du foie et d'autre part de la disparition du tissu adipeux. En effet, puisque le tissu adipeux est fortement réduit après 28 jours de régime CLA2, la lipolyse y est nécessairement faible. On observe ainsi une réduction du taux d'AGL plasmatiques lesquels sont des substrats majeurs de la synthèse hépatique des VLDL $[39,40]$. Une baisse du taux des VLDL plasmatiques associée à leur captage accru par le foie pourrait ainsi être responsable de la diminution de la lipémie induite par le CLA2 (figure 5).

\section{RÉFÉRENCES}

1. COOK ME, MILLER CC, PARK Y, PARIZAM. Immune modulation by altered nutrient metabolism : nutritional control of immune-induced growth depression. Poult Sci 1993; 72: 1301-5.

2. MINER IL, CEDERBERG CA, NIELSEN MK, CHEN $X$, BAlLE CA. Conjugated linoleic acid (CLA), body fat, and apoptosis. Obes Res $2001 ; 9$ : 129-34.

3. BELURY MA. Dietary conjugated linoleic acid in health : physiological effects and mechanisms of action. Annu Rev Nutr 2002 ; 22 : 505-31.

4. MASSO-WELCH PA, ZANGANID, IPC, et al. Isomers of conjugated linoleic acid differ in their effects on angiogenesis and survival of mouse mammary adipose vasculature. / Nutr $2004 ; 134: 299-307$

5. LEE KN, KRITCHEVSKY D, PARIZA MW. Conjugated linoleic acid and atherosclerosis in rabbits. Atherosclerosis $1994 ; 108$ : 19-25.

6. VALEILLE K, GRIPOIS D, BLOUQUIT MF, et al. Lipid atherogenic risk markers can be more favourably influenced by the cis-9, trans-11octadecadienoate isomer than a conjugated linoleic acid mixture or fish oil in hamsters. $\mathrm{Br}$ J Nutr $2004 ; 91: 191-9$.
7. HOUSEKNECHT KL, VANDEN HEUVEL JP, MOYACAMARENA SY, et al. Dietary conjugated linoleic acid normalizes impaired glucose tolerance in the Zucker diabetic fatty fa/fa rat. Biochem Biophys Res Commun $1998 ; 244$ : 678-82

8. BELURY MA, MAHON A, BANNI S. The conjugated linoleic acid (CLA) isomer, t10c12-CLA, is inversely associated with changes in body weight and serum leptin in subjects with type 2 diabetes mellitus. I Nutr 2003; 133 : 257s2605 .

9. TSUBOYAMA-KASAOKA N, TAKAHASHIM TANEMURA K, et al. Conjugated linoleic acid supplementation reduces adipose tissue by apoptosis and develops lipodystrophy in mice. Diabetes 2000 ; 49 : 1534-42.

10. RISERUS U, VESSBY B, ARNLOV J, BASU $S$. Effects of cis-9, trans-11 conjugated linoleic acid supplementation on insulin sensitivity, lipid peroxidation, and proinflammatory markers in obese men. Am / Clin Nutr 2004; 80 : 279-83.

11. OSTROWSKAE, MURALITHARAN M, CROSS RF, BAUMAN DE, DUNSHEA FR. Dietary conjugated linoleic acids increase lean tissue and decrease fat deposition in growing pigs. I Nutr 1999 ; 129 : 2037-42.

12. PARK Y, ALBRIGHT KJ, STORKSON J, LIU W, COOK ME, PARIZA MW. Changes in body composition in mice during feeding and withdrawal of conjugated linoleic acid. Lipids $1999 ; 34: 243-8$

13. SZYMCZYK B, PISULEWSKI PM, SZCZUREK W, HANCZAKOWSKI P. Effects of conjugated linoleic acid on growth performance, feed conversion efficiency, and subsequent carcass quality in broiler chickens. Br / Nutr 2001 ; 85 : 465-73.

14. KOBAK, AKAHOSHIA, YAMASAKIM, et al. Dietary conjugated linolenic acid in relation to CLA differently modifies body fat mass and serum and liver lipid levels in rats. Lipids 2002 ; $37: 343-50$.

15. BLANKSON H, STAKKESTAD IA, FAGERTUN H, THOME, WADSTEIN J, GUDMUNDSEN O. Conjugated linoleic acid reduces body fat mass in overweight and obese humans. J Nutr 2000 ; $130: 2943-8$

16. KELLEY DS, ERICKSON KL. Modulation of body composition and immune cell functions by conjugated linoleic acid in humans and animal models : benefits vs. risks. Lipids 2003; 38 : 377-86.

17. DELANY JP, WEST DB. Changes in body composition with conjugated linoleic acid. / Am Coll Nutr $2000 ; 19$ : 487S-493S.

18. STANGL GI. Conjugated linoleic acids exhibit a strong fat-to-lean partitioning effect, reduce serum VLDL lipids and redistribute tissue lipids in food-restricted rats. / Nutr 2000; 130 : 1140-6.
19. NAGAYOSHI A, MATSUKI $\mathrm{N}$, SAITO $\mathrm{H}$, et al. Defect in assembly process of very-low-density lipoprotein in suncus liver : an animal model of fatty liver. / Biochem (Tokyo) $1995 ; 117$ : 787 93.

20. DEGRACE P, DEMIZIEUX L, GRESTI J, CHARDIGNY IM, SEBEDIO IL, CLOUET P. Association of liver steatosis with lipid oversecretion and hypotriglyceridaemia in C57BL/6j mice fed trans-10, cis-12linoleic acid. FEBS Lett $2003 ; 546$ : 335-9.

21. LIN Y, SCHUURBIERS E, VAN DER VEEN S, DE DECKERE EA. Conjugated linoleic acid isomers have differential effects on triglyceride secretion in Hep G2 cells. Biochim Biophys Acta $2001 ; 1533: 38-46$

22. HO SS, PAL S. Conjugated linoleic acid suppresses the secretion of atherogenic lipoproteins from human HepG2 liver cells. Asia Pac I Clin Nutr $2004 ; 13$ : 570.

23. DE DECKERE EA, VAN AMELSVOORT JM, MCNEILL GP, JONES P. Effects of conjugated linoleic acid (CLA) isomers on lipid levels and peroxisome proliferation in the hamster. $\mathrm{Br} /$ Nutr 1999 ; 82 : 309-17.

24. GAVINO VC, GAVINO G, LEBLANCM], TUCHWEBER $B$. An isomeric mixture of conjugated linoleic acids but not pure cis-9, trans-11octadecadienoic acid affects body weight gain and plasma lipids in hamsters. J Nutr $2000 ; 130: 27-9$.

25. KRITCHEVSKY D, TEPPER S, WRIGHT S, TSO P, CZARNECKI S. Influence of conjugated linoleic acid (CLA) on establishment and progression of atherosclerosis in rabbits. I Am Coll Nutr 2000 ; $19: 4725-4775$.

26. NOONE E), ROCHE HM, NUGENT AP, GIBNEY $\mathrm{M}$ ). The effect of dietary supplementation using isomeric blends of conjugated linoleic acid on lipid metabolism in healthy human subjects. $\mathrm{Br}$ I Nutr 2002 ; 88 : 243-51.

27. SEBEDIO IL, JUANEDA P, DOBSON G, et al. Metabolites of conjugated isomers of linoleic acid (CLA) in the rat. Biochim Biophys Acta $1997 ; 1345: 5-10$.

28. SUGANO M, TSUIITA A, YAMASAKI M, NOGUCHI M, YAMADA K. Conjugated linoleic acid modulates tissue levels of chemical mediators and immunoglobulins in rats. Lipids $1998 ; 33$ : 521-7.

29. DEGRACE P, DEMIZIEUX L, GRESTI J, CHARDIGNY IM, SEBEDIO IL, CLOUET P. Hepatic steatosis is not due to impaired fatty acid oxidation capacities in C57BL/6] mice fed the conjugated trans-10,cis-12isomer of linoleic acid. / Nutr $2004 ; 134$ : 861-7.

30. DEGRACE P, DEMIZIEUX L, GRESTI I, et al. Fatty acid oxidation and related gene expression in heart depleted of carnitine by mildronate treatment in the rat. Mol Cell Biochem $2004 ; 258$ : 171-82. 
31. CLEMENT L, POIRIER H, NIOT I, et al. Dietary trans-10, cis-12 conjugated linoleic acid induces hyperinsulinemia and fatty liver in the mouse. / Lipid Res 2002 ; 43 : 1400-9.

32. GEELEN MJ, HARRIS RA, BEYNEN AC, MCCUNE SA. Short-term hormonal control of hepatic lipogenesis. Diabetes $1980 ; 29: 1006-22$

33. LEWIS GF, CARPENTIER A, ADELI K, GIACCA A Disordered fat storage and mobilization in the pathogenesis of insulin resistance and type 2 diabetes. Endocr Rev 2002 ; 23 : 201-29.

34. CRAIG WY, COOPER AD. Effects of chylomicron remnants and beta-VLDL on the class and composition of newly secreted lipoproteins by HepG2 cells. J Lipid Res 1988 ; 29 : 299-308.
35. WU X, SAKATA N, DIXON J. Ginsberg Hn ExOgenous VLDL stimulates apolipoprotein B secretion from HepG2 cells by both pre- and post-translational mechanisms. I Lipid Res $1994 ; 35: 1200-10$.

36. TACKEN PJ, TEUSINK B, JONG MC, et al. LDL receptor deficiency unmasks altered VLDL triglyceride metabolism in VLDL receptor transgenic and knockout mice. / Lipid Res 2000 ; 41 : 2055-62.

37. OKA K, PASTORE L, KIM IH, et al. Long-term stable correction of low-density lipoprotein receptor-deficient mice with a helperdependent adenoviral vector expressing the very low-density lipoprotein receptor. Circulation $2001 ; 103$ : 1274-81.
38. KOBAYASHI K, OKA K, FORTE T, et al. Reversal of hypercholesterolemia in low density lipoprotein receptor knockout mice by adenovirusmediated gene transfer of the very low density lipoprotein receptor. J Biol Chem 1996 ; 271 : 6852-60.

39. LEWIS GF. Fatty acid regulation of very low density lipoprotein production. Curr Opin Lipidol $1997 ; 8$ : 146-53.

40. HAEMMERLE G, ZIMMERMANN R, STRAUSS $\mathrm{G}$, et al. Hormone-sensitive lipase deficiency in mice changes the plasma lipid profile by affecting the tissue-specific expression pattern of lipoprotein lipase in adipose tissue and muscle. | Biol Chem 2002 ; 277 : 12946-52. 